\title{
Eric M. Genden: reconstruction of the head and neck. A defect-oriented approach, ISBN: 978-1-60406-576-3
}

\author{
Antti A. Mäkitie
}

Published online: 12 March 2013

(c) Springer-Verlag Berlin Heidelberg 2013

This handbook and atlas, published by Thieme in 2012, is edited by Professor Eric M. Genden, MD, FACS, from the Department of Otolaryngology, Head and Neck Surgery, Mount Sinai School of Medicine, New York. The chapters also include contributions of several North-American head and neck surgeons.

A defect-oriented guide is a desired addition and certainly a useful source of surgical knowledge and technical details for both head and neck surgeons and colleagues in training. The chapters cover oral, mandibular, maxillary, cheek, neck, pharyngeal, laryngeal, pharyngo-esophageal, skull base, scalp, paralyzed face and pediatric reconstructions. The ninth chapter is an important review on the pediatric patient population and covers normal development of the upper and lower jaws, donor-site selection, and growth of vascularized bone grafts. The last chapter provides an interesting overview of general aspects and technical details for successful blood vessel management and microvascular free tissue transfer.

Each chapter is organized according to specific defect types of the region in question. The first part of each chapter is devoted to key aspects of relevant anatomy, evaluation and classification of the area. The reader is then guided through the essential reconstructive alternatives. Starting from primary closure and simple local flaps, various reconstructive options are presented. It is noteworthy that only the main flap types with broad evidence in the literature are discussed. Many less cited innovations with limited experience available from case series are not included. Representative clinical case photos and excellent black and white illustrations drawn by Dr. Scott M. Kessler form a prominent part of the book. These also enable the readership to compare the defects of their patients with those presented in the book.

This book provides an excellent framework for successful head and neck reconstruction, and describes the current considerations for assessment of various defects and of the available options for their management. It certainly belongs to the series of handbooks to be used in the daily surgical considerations, which sometimes are complicated. Another important use will be in the surgical education and training of medical students, fellows and other trainees.

\footnotetext{
A. A. Mäkitie $(\square)$

Department of Otolaryngology, Head and Neck Surgery,

Helsinki University Central Hospital and University

of Helsinki, Helsinki, Finland

e-mail: antti.makitie@helsinki.fi
} 